\title{
ON THE ANALOG OF LITTLEWOOD'S PROBLEM IN POWER SERIES FIELDS ${ }^{1}$
}

\author{
R. T. BUMBY
}

If $K$ is any field, we can form the field $\mathfrak{B}_{K}$ whose nonzero elements are expressions

$$
\Xi=\sum_{i=0}^{\infty} a_{i-n} t^{n-i}
$$

with $a_{i-n} \in K, a_{-n} \neq 0$. We let $\Xi^{\prime}$ denote the sum of those terms of $\Xi$ for which $i>n$. Following [3], we write $|\Xi|=e^{n},|0|=0$ and $\|\Xi\|$ $=\left|\Xi^{\prime}\right|$. The main result of $[3]$ is: If $K$ is infinite then there are $\theta$, $\Phi \in \mathfrak{B}_{K}$ such that

$$
|N|\|N \theta\|\|N \Phi\| \geqq e^{-2}
$$

for all polynomials $N \neq 0$. Baker [2] has given definite elements of $\mathfrak{B}_{K}$ for which the left side of (1) is bounded below, but his bound is slightly smaller than $e^{-2}$. We now prove an extension of the theorem of Davenport and Lewis. The present method could also be specialized to give a new proof of their result. First, we prove some lemmas about diophantine approximation in $\mathfrak{B}_{K}$.

Let $M=M\left(d ; c_{1}, \cdots, c_{m}\right)$ be the collection of polynomials $N$ which satisfy

$$
|N| \leqq e^{d}, \quad\left\|N \theta_{i}\right\| \leqq e^{c_{i}} \quad(i=1, \cdots, m)
$$

where $d \geqq 0, c_{i} \leqq-1$. Let $b=\max \left(0, d+m+1+c_{1}+\cdots+c_{m}\right)$.

Lemma 1. $M$ is a $K$-vector space of dimension at least $b$ and at most $d+1$.

Lemma 2. $M(d ; \cdots)=M(d-1 ; \cdots)+M^{\prime}$ where $M^{\prime}$ is either $\{0\}$ or a 1-dimensional space generated by a polynomial of degree $d$.

Proof. When all $c_{i}=-1,(2)$ defines the $(d+1)$-dimensional space of all polynomials of degree at most $d$. Thus Lemma 1 holds in this case. If either $d$ or one of the $c_{i}$ is reduced by 1 , a certain coefficient of $N$ or $N \theta_{i}$ is required to be 0 . This defines a linear subspace of codimension at most 1 in $M$. This observation both proves Lemma 2 and provides the inductive step to prove Lemma 1.

Received by the editors September 14, 1966.

${ }^{1}$ NSF grant No. 4368 and a Rutgers Research Grant have helped to make this note possible. 
REMARK. This proof allows us to construct spaces defined by the inequalities (2) which are 1-dimensional. When $m=1$ the generators of those spaces can be obtained by a continued fraction (cf. [1, \$12]).

Lemma 3. The spaces $M$ all have exactly the dimension $b$ (of Lemma 1) if and only if

$$
|N| \cdot \prod_{i=1}^{m}\left\|N \theta_{i}\right\| \geqq e^{-m}
$$

for all polynomials $N \neq 0$.

Proof. Let $N \neq 0$ be a polynomial. The smallest $M$ containing $N$ (denoted $M(N)$ ) has $b$ such that $e^{b}=|N| \cdot \Pi_{i=1}^{m}\left\|N \theta_{i}\right\| \cdot e^{m+1}$. Also $\operatorname{dim} M(N) \geqq 1$ so if it is exactly equal to $b$ we must have (3). On the other hand, if some $M$ has dimension greater than $b$, the reduction of Lemma 2 leads to a 1-dimensional $M$ for which $b=0$. If $N$ generates this space, it clearly can not satisfy (3).

Corollary. If (3) holds, the $M^{\prime}$ of Lemma 2 is 1-dimensional whenever $b>0$.

THEOREM: If $K$ is infinite, then there is a sequence $\theta_{1}, \theta_{2}, \cdots$ of elements of $\mathfrak{B}_{K}$ such that (3) holds for all polynomials $N \neq 0$ and each $m \geqq 0$. Furthermore, if $\theta_{1}, \cdots, \theta_{m}$ satisfy (3) there is such a sequence that begins with these terms.

Proof. By induction, it suffices to produce $\theta_{m+1}$ when $\theta_{1}, \cdots, \theta_{m}$ are given, since the case $m=0$ is trivially true.

By the proof of Lemma 3 and the inductive hypothesis,

$$
e^{m+1} \cdot|N| \cdot \prod_{i=1}^{m+1}\left\|N \theta_{i}\right\|=e^{b}\left\|N \theta_{m+1}\right\|
$$

where $b=\operatorname{dim} M(N)$. Thus we must show that the coefficients of $t^{-1}, \cdots, t^{-b}$ in $N \theta_{m+1}$ can not all be zero. These coefficients are linear functions on $M(N)$, so we have a linear function from $M(N)$ to $K^{b}$ which we must show to be an isomorphism. If a basis is chosen for $M(N)$ this requires only that a certain determinant be nonzero.

Suppose $\theta_{m+1}=a_{1} t^{-1}+a_{2} t^{-2}+\cdots$. If $N$ has degree $d$, the rule for multiplying elements of $\mathfrak{P}_{K}$ gives the coefficient of $t^{-i}$ in $N \theta_{m+1}$ as a linear combination of $a_{i}, \cdots, a_{d+i}$. Furthermore, $a_{d+i}$ must occur.

If $N_{1}, \cdots, N_{b}$ is a basis for $M$ and $\gamma_{i j}$ is the coefficient of $t^{-i}$ in $N_{j} \theta_{m+1}$, we write $\Delta=\operatorname{det}\left(\gamma_{i j}\right)(1 \leqq i, j \leqq b)$. Lemma 2 and the corollary to Lemma 3 tell us that $M$ has a basis in which $N_{1}, \cdots, N_{b-1}$ are a basis for $M(d-1 ; \cdots)$ and $N_{b}$ has degree $d$. Thus $\gamma_{i j}$ will be a linear 
combination of $a_{1}, \cdots, a_{b+d}$ with $a_{b+d}$ occurring only in $\gamma_{b b}$. A closer look tells us that $\Delta(d ; \cdots)=a_{b+d} \Delta(d-1, \cdots)+($ terms containing $\left.a_{1}, \cdots, a_{b+d-1}\right)$. There are only finitely many choices of $d, c_{1}, \cdots$, $c_{m}$ for which $b+d=k$. Thus if $a_{1}, \cdots, a_{k-1}$ have been obtained such that all $\Delta$ with $b+d<k$ are not zero, there are only a finite number of values of $a_{k}$ which cause any $\Delta$ with $b+d=k$ to vanish. Since $K$ is infinite, we can determine a $\theta_{m+1}$ so that no $\Delta$ is zero. This is all that is required to prove the theorem.

\section{REFERENCES}

1. E. Artin, Quadratische Körper im Gebiet der höheren Kongruenzen. I, Math. Z. 19 (1924), 153-206 (Collected papers, pp. 1-54).

2. A. Baker, On an analog of Littlewood's Diophantine approximation problem, Michigan Math. J. 11 (1964), 247-250.

3. H. Davenport and D. J. Lewis, An analog of a problem of Littlewood, Michigan Math. J. 10 (1963), 157-160.

RUTGERS UNIVERSITY 